\title{
STURM-LIOUVILLE PROBLEM FOR STATIONARY DIFFERENTIAL OPERATOR WITH NONLOCAL INTEGRAL BOUNDARY CONDITION
}

\author{
S. PEČIULYTE் ${ }^{1}$, O. ŠTIKONIENE ${ }^{2}$ and A. ŠTIKONAS ${ }^{2}$ \\ ${ }^{1}$ Vytautas Magnus University \\ ${ }^{1}$ Vileikos 8, LT-44404, Kaunas, Lithuania, \\ ${ }^{2}$ Institute of Mathematics and Informatics \\ ${ }^{2}$ Akademijos 4, LT-08663, Vilnius, Lithuania \\ E-mail: s.peciulyte@if.vdu.lt; osh@fm.vtu.lt; ash@fm.vtu.lt
}

Received May 31 2005; revised November 20, 2005

\begin{abstract}
The Sturm-Liouville problem with various types of nonlocal integral boundary conditions is considered in this paper. In the first part of paper we investigate Sturm-Liouville problem with two cases of nonlocal integral boundary conditions. We prove general properties of the eigenfunctions and eigenvalues for such problem in the complex case. In the second part we investigate real eigenvalues case. The spectrum depends of these problems on boundary condition parameters is analyzed. Qualitative behaviour of all eigenvalues subject to nonlocal boundary condition parameters is described.
\end{abstract}

Key words: Sturm-Liouville problem, nonlocal integral condition

\section{Introduction}

Boundary problems with nonlocal conditions are an area of the fast developing differential equations theory. Problems of this type arise in various fields of physics, biology, biotechnology and etc. Nonlocal conditions come up when value of the function on the boundary is connected with values inside the domain. Theoretical investigation of problems with various type of nonlocal boundary conditions is actual problem and recently it much attention is given in the scientific literature.

A.A. Samarskii and A.V. Bitsadze were originators to such problems. They formulated and investigated nonlocal boundary problem for elliptic equation [1]. J. Canon was one of the pioneers who investigated parabolic problems with integral boundary conditions [4]. Also parabolic problems with nonlocal integral boundary 
conditions were analyzed in $[3,6,7,12,17,20,21]$. Multipoint nonlocal boundary problem for the second order ordinary differential equations was initiated by $\mathrm{V}$. Ilyin and E. Moiseev [10]. Also this problem was investigated in papers [2, 5, 14]. During the last decades the number of differential problems with nonlocal boundary conditions increased significantly.

Quite new area, related with problems of this type, is investigation of the spectrum of differential equations with nonlocal conditions. Eigenvalue problems with nonlocal conditions are closely linked with boundary problems for differential equations with nonlocal conditions $[9,11,13]$. In the papers $[8,16,18]$ the similar problems are investigated for the operators with nonlocal condition of Bitsadze-Samarskii or integral type. Eigenvalue problems for differential operators with nonlocal conditions are considerably less investigated than classical boundary conditions cases.

The purpose of this paper is to analyze a real eigenvalue problem for stationary differential problem with various types of nonlocal boundary conditions. In this paper we analyze stationary problem with two cases of nonlocal integral boundary conditions. We investigate how spectrum of these problems depends on parameters of some nonlocal boundary conditions. Some results are published in paper [19].

In Sect. 2 we analyze Sturm-Liouville problem with nonlocal integral type boundary condition and find general properties of eigenvalues and eigenfunctions in the complex plane, in Sect. 3 we investigate real eigenvalues.

\section{Sturm-Liouville Problem with Nonlocal Integral Type Boundary Condition}

Let us consider Sturm-Liouville problem with one classical boundary condition

$$
\begin{aligned}
& -u^{\prime \prime}=\lambda u, \quad x \in(0,1), \\
& u(0)=0
\end{aligned}
$$

and another nonlocal integral boundary condition:

$$
u(1)=\gamma \int_{0}^{\xi} u(x) \mathrm{d} x \quad(\text { Case } 1)
$$

or

$$
u(1)=\gamma \int_{\xi}^{1} u(x) \mathrm{d} x \quad(\text { Case } 2)
$$

with parameters $\gamma \in \overline{\mathbb{C}}$ and $\xi \in[0,1]$.

Remark 1. For $\gamma=\infty$, we investigate boundary conditions

$$
\int_{0}^{\xi} u(x) \mathrm{d} x=0, \xi>0(\text { Case } 1) \text { and } \int_{\xi}^{1} u(x) \mathrm{d} x=0, \xi<1(\text { Case } 2)
$$

instead boundary conditions (2.3) and (2.4) . 
In the general case eigenvalues $\lambda \in \mathbb{C}$ and eigenfunctions $u(x)$ are the complex functions. We investigate how spectrum depends on boundary condition in the case parameters $\gamma$ and $\xi$.

We note that Case 1 when $\xi=1$ and Case 2 when $\xi=0$ give the same integral boundary condition.

When $\gamma=0$ or $\xi=0$ in problem (2.1)-(2.3) and $\gamma=0$ or $\xi=1$ in problem (2.1)-(2.2), (2.4) we get the problems with classical boundary conditions. Their eigenvalues and eigenfunctions are well-known:

$$
\lambda_{k}=(\pi k)^{2}, \quad u_{k}(x)=\sin (\pi k x), \quad k \in \mathbb{N} .
$$

When $\lambda=0$ then $u(x)=c x$. Substituting this solution into the second boundary condition we get

$$
c=\gamma \int_{0}^{\xi} c x \mathrm{~d} x=c \gamma \frac{\xi^{2}}{2} \quad(\text { Case } 1), \quad c=\gamma \int_{\xi}^{1} c x \mathrm{~d} x=c \gamma \frac{1-\xi^{2}}{2} \quad \text { (Case 2), }
$$

i.e.

$$
c\left(1-\gamma \frac{\xi^{2}}{2}\right)=0 \quad(\text { Case } 1), \quad c\left(1-\gamma \frac{1-\xi^{2}}{2}\right)=0 \quad(\text { Case } 2) .
$$

Nontrivial solutions $(c \neq 0)$ exist if

$$
1-\gamma \frac{\xi^{2}}{2}=0 \quad(\text { Case } 1), \quad 1-\gamma \frac{1-\xi^{2}}{2}=0 \quad(\text { Case } 2) .
$$

Lemma 1. The eigenvalue $\lambda=0$ exists if, and only if $\gamma=\frac{2}{\xi^{2}}$ in Case $1, \gamma=\frac{2}{1-\xi^{2}}$ in Case 2.

In the general case, when $\lambda \neq 0$, eigenfunctions are $u=c \sin (q x)$ and eigenvalues $\lambda=q^{2}$, where $q \in \mathbb{C}_{q} \backslash\{0\}$,

$$
\mathbb{C}_{q}:=\{q \in \mathbb{C} \mid \operatorname{Re} q>0 \text { or } \operatorname{Re} q=0, \operatorname{Im} q>0 \text { or } q=0\} .
$$

They satisfy equation (2.1), boundary condition (2.2) and nonlocal boundary condition (2.3) or nonlocal boundary condition (2.4).

If $\lambda \neq 0$, the nonlocal boundary condition is satisfied, when

$c \sin q=c \gamma \int_{0}^{\xi} \sin (q x) \mathrm{d} x \quad($ Case 1$), \quad c \sin q=c \gamma \int_{\xi}^{1} \sin (q x) \mathrm{d} x \quad$ (Case 2),

i.e.

$c\left(\sin q-\gamma \frac{1-\cos (\xi q)}{q}\right)=0($ Case 1$), c\left(\sin q-\gamma \frac{\cos (\xi q)-\cos q}{q}\right)=0$ (Case 2).

So, a nontrivial solution exists if $q(q \neq 0)$ is a root of equation

$$
\begin{aligned}
& f_{1}(q):=2 \gamma \frac{\sin ^{2} \frac{\xi q}{2}}{q^{2}}-\frac{\sin q}{q}=0 \quad(\text { Case } 1), \\
& f_{2}(q):=2 \gamma \frac{\sin \frac{(1+\xi) q}{2} \sin \frac{(1-\xi) q}{2}}{q^{2}}-\frac{\sin q}{q}=0 \quad(\text { Case } 2) .
\end{aligned}
$$


If $\sin q=0$ and $\sin \frac{\xi q}{2}=0$ in Case 1 , and $\sin \frac{(1+\xi) q}{2}=0$ or $\sin \frac{(1-\xi) q}{2}=0$ and $\sin q=0$ in Case 2, then equation (2.6) or (2.7) is valid for all $\gamma \in \mathbb{C}$. In this case we get constant eigenvalues, which don't depend on parameter $\gamma$. If parameter $\xi$ is an irrational number, then such eigenvalues don't exist.

Let $\xi=r=\frac{m}{n} \in \mathbb{Q}$. For $\xi \in(0,1)$ we suppose that $m$ and $n(n>m>0)$ are positive coprime integer numbers. If $\xi=0$, we suppose $m=0, n=1$ and if $\xi=1$, we suppose $m=1, n=1$. Let denote subset $\mathbb{N}_{m}:=\{n \in \mathbb{N} \mid n=k m, k \in \mathbb{N}\}$ of integer positive numbers, $\mathbb{N}_{e}=\left\{k \in \mathbb{N}_{2} \mid k \leq n\right\} \cup\{0\}$ are even numbers and $\mathbb{N}_{o}=\left\{k \in \mathbb{N} \backslash \mathbb{N}_{2} \mid k \leq n\right\}$ are odd numbers.

Lemma 2. Constant eigenvalues exist only for rational numbers $\xi=\frac{m}{n} \in[0,1]$, and those eigenvalues are given by: $\lambda_{k}=(n \pi k)^{2}, k \in \mathbb{N}, m \in \mathbb{N}_{e}$ and $\lambda_{k}=$ $(2 n \pi k)^{2}, k \in \mathbb{N}, m \in \mathbb{N}_{o}$ in Case $1 ; \lambda_{k}=(n \pi k)^{2}, k \in \mathbb{N}, n-m \in \mathbb{N}_{e}$ and $\lambda_{k}=(2 n \pi k)^{2}, k \in \mathbb{N}, n-m \in \mathbb{N}_{o}$ in Case 2 .

Proof. Let us define functions $S_{j}(z)=\frac{\sin (j z)}{\sin z}, j \in \mathbb{N} \cup\{0\}$. When $j \geq 2$, we can express them by the Moivre formula:

$$
\begin{aligned}
& S_{2 k}(z)=2 k \cos ^{2 k-1} z-\left(\begin{array}{c}
2 k \\
3
\end{array}\right) \cos ^{2 k-3} z \sin ^{2} z+\ldots+(-1)^{k-1} 2 k \cos z \sin ^{2 k-2} z, \\
& S_{2 k+1}(z)=(2 k+1) \cos ^{2 k} z-\left(\begin{array}{c}
2 k+1 \\
3
\end{array}\right) \cos ^{2 k-2} z \sin ^{2} z+\ldots+(-1)^{k} \sin ^{2 k} z .
\end{aligned}
$$

We see, that $S_{1}(z) \equiv 1, S_{0}(z) \equiv 0$ and for $j \geq 2$ functions $S_{j}(z)$ are entire transcendental with the first order of growth. Functions $\sin z$ and $S_{j}(z)$ don't have common zeroes. Then common zeroes of functions $\sin m z$ and $\sin n z$ are zeroes of function $\sin z$ if $0<m<n$. Therefore in this case, functions $S_{n}(z)$ and $S_{m}(z)$ don't have common zeroes.

In Case 1 from equation (2.6) we get:

$$
\begin{aligned}
& \sin \frac{q}{n} \cdot\left[\frac{2 \gamma}{q} S_{m / 2}^{2}\left(\frac{q}{n}\right) \sin \frac{q}{n}-S_{n}\left(\frac{q}{n}\right)\right]=0, m \in \mathbb{N}_{e}, \\
& \sin \frac{q}{2 n} \cdot\left[\frac{2 \gamma}{q} S_{m}^{2}\left(\frac{q}{2 n}\right) \sin \frac{q}{2 n}-S_{2 n}\left(\frac{q}{2 n}\right)\right]=0, m \in \mathbb{N}_{o} .
\end{aligned}
$$

In Case 2 from equation (2.7) we get:

$$
\begin{aligned}
& \sin \frac{q}{n} \cdot\left[\frac{2 \gamma}{q} S_{(n+m) / 2}\left(\frac{q}{n}\right) S_{(n-m) / 2}\left(\frac{q}{n}\right) \sin \frac{q}{n}-S_{n}\left(\frac{q}{n}\right)\right]=0, n-m \in \mathbb{N}_{e}, \\
& \sin \frac{q}{2 n} \cdot\left[\frac{2 \gamma}{q} S_{n+m}\left(\frac{q}{2 n}\right) S_{n-m}\left(\frac{q}{2 n}\right) \sin \frac{q}{2 n}-S_{2 n}\left(\frac{q}{2 n}\right)\right]=0, n-m \in \mathbb{N}_{o} .
\end{aligned}
$$

We note that numbers $n-m$ and $n+m$ are both odd or both even.

If $\sin \frac{q}{n}=0$, we have constant eigenvalues for $m \in \mathbb{N}_{e}$ in Case 1 and $n-m \in \mathbb{N}_{e}$ in Case 2. From this equation we get that $q=n \pi k, k \in \mathbb{N}$, so $\lambda_{k}=(n \pi k)^{2}, k \in \mathbb{N}$. If $\sin \frac{q}{2 n}=0$, we have constant eigenvalues for $m \in \mathbb{N}_{o}$ (Case 1) and odd $n-m \in \mathbb{N}_{o}$ (Case 2). Therefore, $q_{k}=2 n \pi k, k \in \mathbb{N}$ in Case 1 and $\lambda_{k}=(2 n \pi k)^{2}, k \in \mathbb{N}$ in Case 2. 
Remark 2. Functions $S_{j}(z)=P_{j}(\cos z)$, where $P_{j}$ is the polynomial (with real integer coefficients) and $\operatorname{deg} P_{j}=j-1, j>1$ :

$$
\begin{aligned}
& \quad P_{2 k}(z)=2 k z^{2 k-1}-\left(\begin{array}{c}
2 k \\
3
\end{array}\right) z^{2 k-3}\left(1-z^{2}\right)+\cdots+(-1)^{k-1} 2 k z\left(1-z^{2}\right)^{k-1}, \\
& \quad P_{2 k+1}(z)=(2 k+1) z^{2 k}-\left(\begin{array}{c}
2 k+1 \\
3
\end{array}\right) z^{2 k-2}\left(1-z^{2}\right)+\cdots+(-1)^{k}\left(1-z^{2}\right)^{k}, \\
& \text { and } P_{0} \equiv 0, P_{1} \equiv 1 .
\end{aligned}
$$

Remark 3. When $n=1$ ( $m=0$ in Case 1 or $m=1$ in Case 2 ) we have only constant eigenvalues.

Let us analyze non constant eigenvalues. We define functions:

$$
\begin{aligned}
& f_{1 r}(z):=2 \gamma P_{m / 2}^{2}\left(\cos \frac{z}{n}\right) \frac{\sin \frac{z}{n}}{z}-P_{n}\left(\cos \frac{z}{n}\right), 0<m \in \mathbb{N}_{e}, \\
& f_{1 r}(z):=2 \gamma P_{m}^{2}\left(\cos \frac{z}{2 n}\right) \frac{\sin \frac{z}{2 n}}{z}-P_{2 n}\left(\cos \frac{z}{2 n}\right), m \in \mathbb{N}_{o}
\end{aligned}
$$

in Case 1, and

$$
\begin{aligned}
f_{2 r}(z) & :=2 \gamma P_{\frac{n+m}{2}}\left(\cos \frac{z}{n}\right) P_{\frac{n-m}{2}}\left(\cos \frac{z}{n}\right) \frac{\sin \frac{z}{n}}{z}-P_{n}\left(\cos \frac{z}{n}\right)=0, \\
0 & <n-m \in \mathbb{N}_{e}, \\
f_{2 r}(z) & :=2 \gamma P_{n+m}\left(\cos \frac{z}{2 n}\right) P_{n-m}\left(\cos \frac{z}{2 n}\right) \frac{\sin \frac{z}{2 n}}{z}-P_{2 n}\left(\cos \frac{z}{2 n}\right)=0, \\
n & -m \in \mathbb{N}_{o}
\end{aligned}
$$

in Case 2.

Remark 4. In the case $\gamma=\infty$ we define functions:

$$
\begin{aligned}
f_{1 r}(z) & :=2 P_{m / 2}^{2}\left(\cos \frac{z}{n}\right) \frac{\sin \frac{z}{n}}{z}, 0<m \in \mathbb{N}_{e} ; \\
f_{1 r}(z) & :=2 P_{m}^{2}\left(\cos \frac{z}{2 n}\right) \frac{\sin \frac{z}{2 n}}{z}, m \in \mathbb{N}_{o} \\
f_{2 r}(z) & :=2 P_{\frac{n+m}{2}}\left(\cos \frac{z}{n}\right) P_{\frac{n-m}{2}}\left(\cos \frac{z}{n}\right) \frac{\sin \frac{z}{n}}{z}, 0<n-m \in \mathbb{N}_{e} ; \\
f_{2 r}(z) & :=2 P_{n+m}\left(\cos \frac{z}{2 n}\right) P_{n-m}\left(\cos \frac{z}{2 n}\right) \frac{\sin \frac{z}{2 n}}{z}-P_{2 n}\left(\cos \frac{z}{2 n}\right)=0, \\
n & -m \in \mathbb{N}_{o} .
\end{aligned}
$$

Lemma 3. There is countable number of eigenvalues, which depend on parameter $\gamma$, for every $\gamma \in \overline{\mathbb{C}}$ and every $\xi \in(0,1]$ in Case 1 and every $\xi \in[0,1)$ in Case 2. Point $\lambda=\infty$ is accumulation point of those eigenvalues.

Proof. Functions $f_{k}(\sqrt{\lambda}), k=1,2$ for irrational $\xi$ and functions $f_{k r}(\sqrt{\lambda}), k=1,2$ for rational $\xi=r=m / n$ are entire transcendental functions with order of growth 
equal to $\frac{1}{2}$. Such functions get every $\gamma$-value infinity (countable) times, and $\lambda=\infty$ is an accumulation point of $\gamma$-values (see, [15]).

For instance, functions $f_{i}(\sqrt{\lambda}), f_{i r}(\sqrt{\lambda})$ have infinity number of zeroes points. We can get all non constant eigenvalues (which depend on parameter $\gamma$ ) as $\gamma$-values of meromorphic functions defined on the set $\mathbb{C}_{q}$ :

$$
\begin{aligned}
& \gamma_{1}(q):=\frac{q \sin q}{1-\cos (\xi q)}=\frac{q \sin q}{2 \sin ^{2}(\xi q / 2)}, \quad \text { for } \xi \in(0,1), \xi \notin \mathbb{Q}, \\
& \gamma_{1 r}(q):= \begin{cases}\frac{q P_{n}\left(\cos \left(\frac{q}{n}\right)\right)}{2 \sin \left(\frac{q}{n}\right) P_{\frac{m}{2}}^{2}\left(\cos \left(\frac{q}{n}\right)\right)}, \quad 0<m \in \mathbb{N}_{e}, \\
\frac{q P_{2 n}\left(\cos \left(\frac{q}{2 n}\right)\right)}{2 \sin \left(\frac{q}{2 n}\right) P_{m}^{2}\left(\cos \left(\frac{q}{2 n}\right)\right)}, m \in \mathbb{N}_{o},\end{cases}
\end{aligned}
$$

in Case 1, and

$$
\begin{aligned}
& \gamma_{2}(q):=\frac{q \sin q}{2 \sin ((1+\xi) q / 2) \sin ((1-\xi) q / 2)}, \quad \text { for } \xi \in(0,1), \xi \notin \mathbb{Q}, \\
& \gamma_{2 r}(q):= \begin{cases}\frac{q P_{n}\left(\cos \left(\frac{q}{n}\right)\right)}{2 \sin \left(\frac{q}{n}\right) P_{\frac{n+m}{2}}\left(\cos \left(\frac{q}{n}\right)\right) P_{\frac{n-m}{2}}\left(\cos \left(\frac{q}{n}\right)\right)}, & 0<n-m \in \mathbb{N}_{e}, \\
\frac{q P_{2 n}\left(\cos \left(\frac{q}{2 n}\right)\right)}{2 \sin \left(\frac{q}{2 n}\right) P_{n+m}\left(\cos \left(\frac{q}{2 n}\right)\right) P_{n-m}\left(\cos \left(\frac{q}{2 n}\right)\right)}, & n-m \in \mathbb{N}_{o},\end{cases} \\
& \text { for } \xi=r \in \mathbb{Q}(0,1) \text {, }
\end{aligned}
$$

in Case 2. We can use functions $\gamma_{k}$ for rational $\xi$ too, but in this case the constant eigenvalues points are isolated singular points.

Remark 5. The poles of the functions $\gamma_{1}(q), \gamma_{2}(q), \gamma_{1 r}(q), \gamma_{2 r}(q)$ are eigenvalues of the problem (2.1)-(2.3) or the problem (2.1)-(2.2),(2.4) in the case $\gamma=\infty$.

The graphics of functions $\left|\gamma_{1 r}(z / \pi)\right|$ and $\left|\gamma_{2 r}(z / \pi)\right|$ for various rational $\xi$ are presented in the Fig. 1. As $\overline{\cos z}=\cos \bar{z}, \overline{\sin z}=\sin \bar{z}$, and polynomials $P_{n}$ have real coefficients, we get similar property for functions $\gamma_{k}$ and $\gamma_{k r}: \overline{\gamma(z)}=\gamma(\bar{z})$, $\overline{\gamma_{r}(z)}=\gamma_{r}(\bar{z})$. So, graphics are drawn only for $\operatorname{Im} z \geq 0$ and $\operatorname{Re} z \geq 0$.

Proposition 1. All zeroes and poles of meromorphic functions $\gamma_{1 \xi}, \gamma_{1 r}, \gamma_{2 \xi}$, $\gamma_{2 r}$ lie on the positive part of the real axis.

Proof. The proof directly follows from (2.8) and (2.10) and properties of sinus function (all zeroes of this function are real numbers $q=k \pi, k \in \mathbb{N}$ ). So, on the $\mathbb{C}_{q}$ we have only positive zeroes and poles.

Remark 6. If $\xi$ is a irrational number, then $z_{j}=\pi j, j \in \mathbb{N}$ are zeroes of those functions $\gamma_{1}, \gamma_{2}$. The points $p_{k}=2 \pi k / \xi, k \in \mathbb{N}$ are the second order poles in Case 1 (see, formula (2.8)), and points $p_{k}=2 \pi k /(1+\xi), k \in \mathbb{N}$ and $\tilde{p}_{l}=2 \pi l /(1-\xi)$, $l \in \mathbb{N}$ are the first order poles in Case 2 (see, formula (2.10)). 


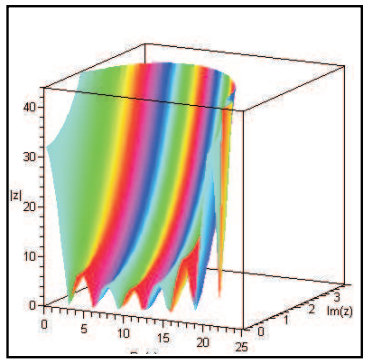

Case $1, \xi=\frac{1}{4}$

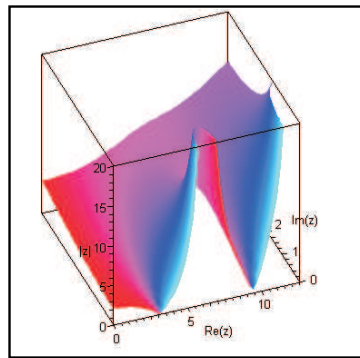

Case $1, \xi=1$; Case $2, \xi=0$

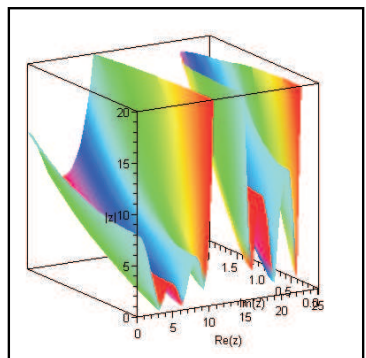

Case $1, \xi=\frac{1}{2}$

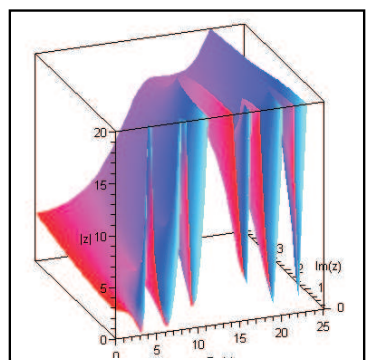

Case $2, \xi=\frac{1}{2}$

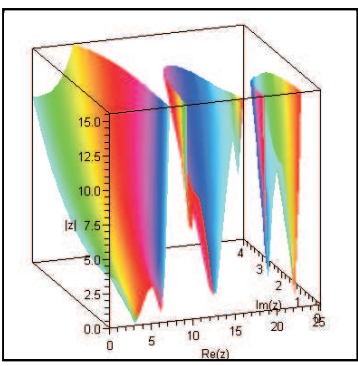

Case $1, \xi=\frac{3}{4}$

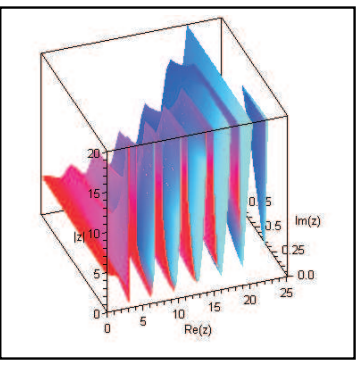

Case $2, \xi=\frac{3}{4}$

Figure 1. Functions $\left|\gamma_{1 r}(z / \pi)\right|$ and $\left|\gamma_{2 r}(z / \pi)\right|$ for various $\xi$.

Remark 7. When $\xi=r=m / n \in \mathbb{Q}$, then a part of zeroes $z_{j}$ of functions $\gamma_{1}, \gamma_{2}$ coincide with the poles $p_{k}$ or $p_{l}$. In Case 1 points $p_{k}=\pi k / m, k \in \mathbb{N}_{2 n}$ are poles. They are poles of the second order, except $k \in \mathbb{N}_{m n}, m \in \mathbb{N}_{e}$ and $k \in \mathbb{N}_{2 m n}, m \in$ $\mathbb{N}_{o}$ (coincide with the case of constant eigenvalues) which are the first order poles. When $m=1$ and $m=2$ there aren't poles of the second order. In Case 2 if pair $(n, m)$ are coprime numbers then pairs $(m+n, m-n),(m+n, n),(m-n, n)$ are also coprime numbers. We have two families of the poles: $p_{k}=\pi k /(n+m)$, $k \in \mathbb{N}_{2 n}$, and $\tilde{p}_{l}=\pi l /(n-m), l \in \mathbb{N}_{2 n}$. The poles from the first family coincide with the poles from the second family in the points $q_{i}=\pi i, i \in \mathbb{N}_{n}, n-m \in \mathbb{N}_{e}$ or $q_{j}=\pi j, j \in \mathbb{N}_{2 n}, n-m \in \mathbb{N}_{o}$. These points are zeroes of sinus function too (they coincide with the case of constant eigenvalues). So, in Case 2 all points $p_{k}, k \in \mathbb{N}_{2 n}$ or $\tilde{p}_{l}, l \in \mathbb{N}_{2 n}$ are poles of the first order.

Remark 8. We can enumerate all poles $p_{k}, k \in \mathbb{N}$ in the increasing order, i.e. $p_{1}<$ $p_{2}<\cdots<p_{k}=p_{k+1}<\ldots$ There $p_{k}$ is the first order pole which coincide with constant eigenvalue point. Formally we note $p_{0}=0$.

From inequalities $\sinh |\operatorname{Im} q| \leq|\sin q| \leq \cosh (\operatorname{Im} q)$ we get estimates 


$$
\begin{aligned}
& \frac{|q| \sinh |\operatorname{Im} q|}{2 \cosh ^{2}(\xi \operatorname{Im} q / 2)} \leq\left|\gamma_{1}\right| \leq \frac{|q| \cosh (\operatorname{Im} q)}{2 \sinh ^{2}|\xi \operatorname{Im} q / 2|}, \\
& \frac{|q| \sinh |\operatorname{Im} q|}{2 \cosh ((1-\xi) \operatorname{Im} q / 2) \cosh ((1-\xi) \operatorname{Im} q / 2)} \leq\left|\gamma_{2}\right| \\
& \quad \leq \frac{|q| \cosh (\operatorname{Im} q)}{2 \sinh |(1+\xi) \operatorname{Im} q / 2| \sinh |(1-\xi) \operatorname{Im} q / 2|} .
\end{aligned}
$$

Corollary 1. The following limits are valid: $\lim _{q \rightarrow \pm \infty} \gamma_{k}=\infty, \quad k=1,2$.

\section{Real Eigenvalues Case}

Let us consider the case when parameter $\gamma \in \mathbb{R}$. Then we investigate Sturm-Liouville problem (2.1)-(2.3) or (2.1)-(2.2), (2.4) with real eigenfunctions and real eigenvalues $\lambda \in \mathbb{R}$.

Instead of $q \in \mathbb{C}_{q}$ we take $q$ only on rays $q=x \geq 0$ and $q=-\mathrm{i} x, x \leq 0$. We have positive eigenvalues in the case of ray $q=x>0$ and negative eigenvalues in the case of ray $q=-\mathrm{i} x, x<0$. Point $q=x=0$ corresponds to $\lambda=0$. For function $f: \mathbb{C}_{q} \rightarrow \mathbb{C}$ we have two restrictions on those rays: $f_{+}(x)=f(x+\mathrm{i} 0)$ for $x \geq 0$ and $f_{-}(x)=f(0-\mathrm{i} x)$ for $x \leq 0$. Function $f_{+}$corresponds to the positive eigenvalues case and function $f_{-}$to the negative eigenvalues case. We investigate all real eigenvalues

$$
\lambda_{k}=\left\{\begin{array}{rl}
x_{k}^{2}, \text { for } x_{k} & \geq 0, \\
-x_{k}^{2}, \text { for } x_{k} & \leq 0
\end{array} \quad k \in \mathbb{N},\right.
$$

using a function $f: \mathbb{R} \rightarrow \mathbb{C}$ :

$$
f(x)=\left\{\begin{array}{l}
f_{+}(x), \text { for } x \geq 0 \\
f_{-}(x), \text { for } x \leq 0
\end{array}\right.
$$

For complex functions (2.8) and (2.10) these functions are real and can be written as:

$$
\begin{aligned}
& \gamma_{1}(x):= \begin{cases}\gamma_{1-}(x)=\frac{x \sinh x}{2 \sinh ^{2}\left(\frac{\xi x}{2}\right)}, \text { for } x \leq 0, \\
\gamma_{1+}(x)=\frac{x \sin x}{2 \sin ^{2}\left(\frac{\xi x}{2}\right)}, \quad \text { for } x \geq 0 ;\end{cases} \\
& \gamma_{2}(x):= \begin{cases}\gamma_{2-}(x)=\frac{x \sinh x}{2 \sinh \left(\frac{(1+\xi) x}{2}\right) \sinh \left(\frac{(1-\xi) x}{2}\right)} & \text { for } x \leq 0, \\
\gamma_{2+}(x)=\frac{x \sin x}{2 \sin \left(\frac{(1+\xi) x}{2}\right) \sin \left(\frac{(1-\xi) x}{2}\right)} & \text { for } x \geq 0 .\end{cases}
\end{aligned}
$$

We use notation $f(x ; \xi)$ or $f(x)$ when investigate function $f(x, \xi)$ as one dimensional with fixed parameter $\xi$. Graphics of functions $\gamma_{1}(x ; \xi)$ and $\gamma_{2}(x ; \xi)$ for various $\xi$ are shown in Fig. 2. 


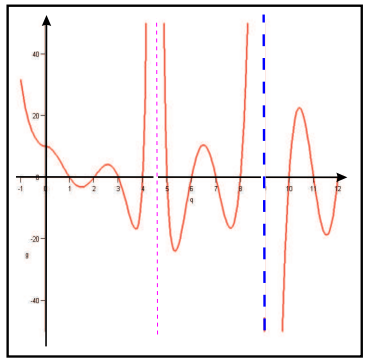

Case $1, \xi=\frac{4}{9}$

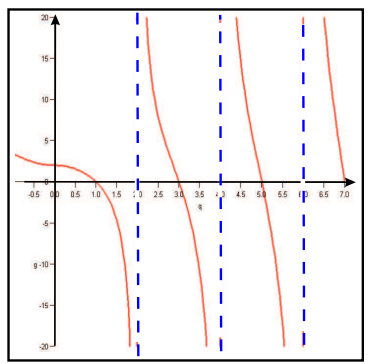

Case $1, \xi=1$; Case $2, \xi=0$

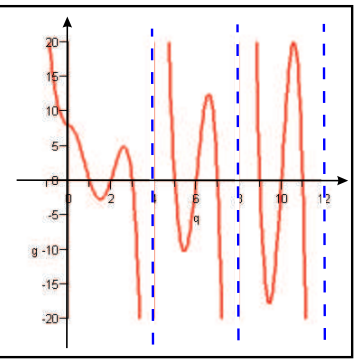

Case $1, \xi=\frac{1}{2}$

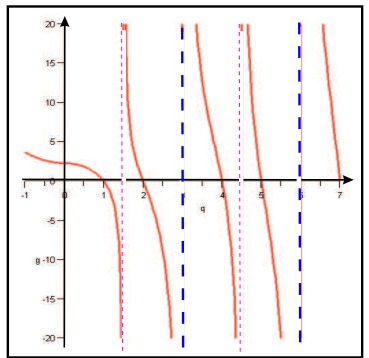

Case $2, \xi=\frac{1}{3}$

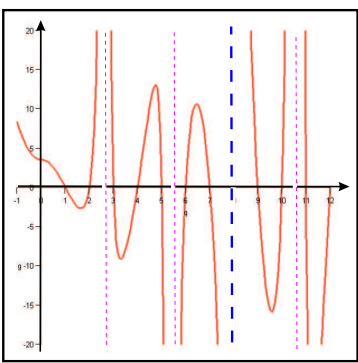

Case $1, \xi=\frac{3}{4}$

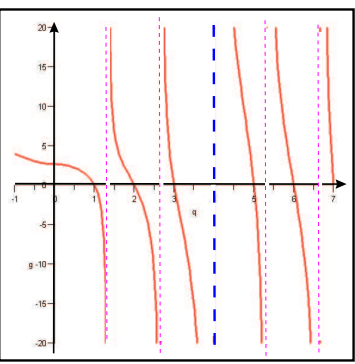

Case $2, \xi=\frac{1}{2}$

Figure 2. Functions $\gamma_{1}(x / \pi ; \xi)$ and $\gamma_{2}(x / \pi ; \xi)$.

Let's enumerate all poles $p_{k}, k \in \mathbb{N}$ in the increasing order (see, Remark 8 in Sect. 2). Functions $\gamma_{1+}(x)$ and $\gamma_{2+}(x)$ are defined in intervals $(\alpha, \beta)=\left(p_{k-1}, p_{k}\right)$, $k \in \mathbb{N}$, where $p_{k-1}<p_{k}$.

Proposition 2. Functions $\gamma_{1-}(x ; \xi)$ and $\gamma_{2-}(x ; \xi)$ are monotone decreasing functions for $x<0$ and all $\xi \in(0,1)$ and for $\xi=0$ in Case $2(\xi=1$ in Case 1). Function $\gamma_{2+}(x ; \xi)$ is monotone decreasing function in each interval $(\alpha, \beta)$ for all $\xi \in(0,1)$ and for $\xi=0$ in Case $2(\xi=1$ in Case 1$)$.

Proof. Functions $\gamma_{1-}(x)$ and $\gamma_{2-}(x)$ are even, when $x \in \mathbb{R}$, and $\gamma_{1-}(0)=\frac{2}{\xi^{2}}$, $\gamma_{2-}(0)=\frac{2}{1-\xi^{2}}$ and $\gamma_{1-}(+\infty)=\gamma_{2-}(+\infty)=+\infty$. Therefore, we need to show that in interval $(0,+\infty)$ these functions are increasing.

Let's consider function $y_{1}(x):=x \operatorname{coth} x, x>0$. It is evident that $\sinh x>x$. So,

$$
y_{1}^{\prime}(x)=\frac{\sinh (2 x)-2 x}{2 \sinh ^{2} x}>0,
$$

and $y_{1}(x)$ is increasing positive function. Then $1 / y_{1}(x)=\frac{1}{x} \tanh x$ is decreasing positive function and it's derivative is negative.

Let's consider function $y(\xi, x):=\frac{1}{\xi} \tanh (\xi x)-\tanh x, x>0$ and $\xi \in(0,1)$. For this function

$$
\lim _{\xi \rightarrow 0+} y(\xi ; x)=x-\tanh x>0, \quad \lim _{\xi \rightarrow 1-} y(\xi ; x)=0
$$


for all $x>0$. Its derivative with respect to $\xi$

$$
y^{\prime}(\xi ; x)=\left(\frac{1}{\xi} \tanh (\xi x)\right)^{\prime}=x\left(\frac{1}{\xi x} \tanh (\xi x)\right)^{\prime}<0 .
$$

So, $y(\xi ; x)$ is monotone decreasing function in $(0,1)$ and from $(3.3)$ we have that $y(\xi ; x)>0$ for all $\xi \in(0,1)$ and all $x>0$.

Let's consider function $y_{2}(x, \xi):=\frac{\sinh x}{\sinh (\xi x)}, x>0$. Its derivative

$$
y_{2}^{\prime}(x ; \xi)=\frac{\cosh x \sinh (\xi x)-\xi \cosh (\xi x) \sinh x}{\sinh ^{2}(\xi x)}=\frac{\xi y(\xi, x) \cosh x \cosh (\xi x)}{\sinh ^{2}(\xi x)}>0 .
$$

So, $y_{2}(x, \xi)$ is increasing positive function for all $x>0$ and $\xi \in(0,1)$.

Function

$$
\gamma_{1-}(x ; \xi)=2 \frac{\frac{x}{2} \cosh \frac{x}{2}}{\sinh \frac{x}{2}}\left(\frac{\sinh \frac{x}{2}}{\sinh \left(\frac{\xi x}{2}\right)}\right)^{2}=2 y_{1}\left(\frac{1}{2} x\right) \cdot y_{2}\left(\frac{1}{2} x ; \xi\right) \cdot y_{2}\left(\frac{1}{2} x ; \xi\right)
$$

is monotone increasing function for $x>0$ as product of monotone increasing positive functions. For $\xi=1$ function $y_{2} \equiv 1$, and proposition is valid in this case too.

Function

$$
\begin{aligned}
\gamma_{2-}(x ; \xi) & =\frac{x}{2} \operatorname{coth}\left(\frac{(1-\xi) x}{2}\right)+\frac{x}{2} \operatorname{coth}\left(\frac{(1+\xi) x}{2}\right) \\
& =\frac{y_{1}\left(\frac{1-\xi}{2} x\right)}{1-\xi}+\frac{y_{1}\left(\frac{1+\xi}{2} x\right)}{1+\xi}
\end{aligned}
$$

is monotone increasing positive function for $x>0$ as sum of monotone increasing positive functions.

Let's consider function $y_{3}(x):=x \cot x, x>0, x \neq k \pi, k \in \mathbb{N}$. It is evident that $\sin x<x$. So,

$$
y_{3}^{\prime}(x)=\frac{\sin (2 x)-2 x}{2 \sin ^{2} x}<0,
$$

and $y_{3}(x)$ is monotone decreasing function in the intervals $(\pi(k-1), \pi k), k \in \mathbb{N}$. Function

$$
\begin{aligned}
\gamma_{2+}(x ; \xi) & =\frac{x}{2} \cot \left(\frac{(1-\xi) x}{2}\right)+\frac{x}{2} \cot \left(\frac{(1+\xi) x}{2}\right) \\
& =\frac{y_{3}\left(\frac{1-\xi}{2} x\right)}{1-\xi}+\frac{y_{3}\left(\frac{1+\xi}{2} x\right)}{1+\xi}
\end{aligned}
$$

is decreasing function (as sum of decreasing functions) in each interval $(\alpha, \beta)$. For $\xi=0$ function $\gamma_{2+}(x ; 0)=2 y_{3}\left(\frac{x}{2}\right)$, and proposition is valid in this case too.

In the Sect. 2 we show that $\lambda=0$ exists if and only if $\gamma=\gamma_{0}$ (see, Lemma 1), and $\gamma_{0}=\frac{2}{\xi^{2}}, \xi \in(0,1]$ in Case $1, \gamma_{0}=\frac{2}{1-\xi^{2}}, \xi \in[0,1)$ in Case 2. Now from Proposition 2 we will derive a few results for eigenvalues. 
Lemma 4. For $\gamma>\gamma_{0}$ one negative eigenvalue exists, and for $\gamma \leq \gamma_{0}$ there aren't negative eigenvalues.

Proof. Functions $\gamma_{1-}(x)$ and $\gamma_{2-}(x)$ are monotone decreasing functions when $x<$ $0, \gamma_{1-}(-\infty)=\gamma_{2-}(-\infty)=+\infty$ and $\gamma_{1-}(0)=\frac{2}{\xi^{2}}, \gamma_{2-}(0)=\frac{2}{1-\xi^{2}}$. Therefore, equation $\gamma=\gamma_{k-}(x), k=1,2$ has negative root only for $\gamma>\gamma_{0}$.

Lemma 5. All eigenvalues of the problem (2.1)-(2.2), (2.4) with real $\gamma$ are real. Each positive eigenvalue $\lambda_{k}(\gamma)=x_{k}^{2}(\gamma)$, where $x_{k} \in\left(p_{k-1}, p_{k}\right)$ if $p_{k-1}<p_{k}$ or $x_{k}=p_{k}$ if $p_{k-1}=p_{k}$.

Proof. The proof follows from Proposition 2 for the function $\gamma_{2}$ in the case of non constant eigenvalues, because all constant eigenvalues are real and positive

Remark 9. We enumerate eigenvalues $\lambda_{k}(\gamma)=x_{k}^{2}(\gamma)$ as $x_{k}(0)=\pi k$, i.e. using the case of classical boundary condition.

In Case 2 of the boundary conditions we prove the following asymptotical properties of eigenvalues.

Corollary 2. For the problem (2.1)-(2.2), (2.4) the properties

$\lim _{\gamma \rightarrow-\infty} x_{k}(\gamma)=p_{k}, \quad \lim _{\gamma \rightarrow+\infty} x_{k}(\gamma)=p_{k-1}, \quad k \in \mathbb{N} \backslash\{1\}, \quad \lim _{\gamma \rightarrow+\infty} x_{1}(\gamma)=-\infty$

are valid.

In Case 1 of the boundary conditions there isn't such simple spectrum. In this case for real $\gamma$ can exist multiple and complex eigenvalues. In many cases it is necessary to know when all eigenvalues are positive and non multiple, i.e. the spectrum of analyzed problem is the same as for the classical problem. When the qualitative root distribution depends on parameters $\gamma$ and $\xi$, it is necessary to find such interval for $\gamma$ where the spectrum of the problem satisfies this property.

Graphics of functions $h_{1}(x):=\sin x+x \cos x, h_{2}(x):=1-\cos x-x \sin x$ for $x \geq 0$ are given in Fig. 3. Let us suppose that $x_{1}, x_{2}, x_{3}$ are the first three positive zeroes of function $h_{1}$ and $z_{1}, z_{2}, z_{3}, z_{4}$ are the first four positive zeroes of function $h_{2}$. We define $\xi_{k}:=\pi / x_{k}$ and $\gamma_{k}:=x_{k} \sin x_{k} / 2$. Then $x_{1} \approx 2.02876$, $x_{2} \approx 4.91318, x_{3} \approx 7.977, \xi_{1} \approx 1.54853, \xi_{2} \approx 0.639421, \xi_{3} \approx 0.393743, \gamma_{1} \approx$ $0.90985, \gamma_{2} \approx-2.4072, \gamma_{3} \approx 3.95836, z_{1} \approx 2.3311, z_{2}=2 \pi, z_{3} \approx 9.2084$, $z_{4}=4 \pi$.

Lemma 6. If $\gamma_{2} \leq \gamma \leq \gamma_{3}$, then all eigenvalues of the problem (2.1)-(2.3) are real for all $\xi \in(0,1)$, and limitary cases are realizable when $\xi=\xi_{2}$ and $\xi=\xi_{3}$. If $\gamma_{2}<\gamma \leq 2$, then all eigenvalues are positive and simple for all $\xi \in(0,1)$.

Proof. We can consider only non constant eigenvalues, because the constant eigenvalues (if they exist) are positive. Function $\gamma_{1+}$ defines distribution of positive eigenvalues. We resolve $\gamma_{1+}$ in multiplicands: 


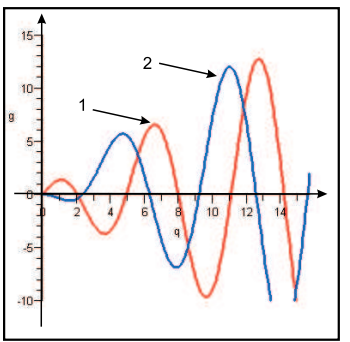

Figure 3. Functions $h_{1}(1)$ and $h_{2}(2)$.

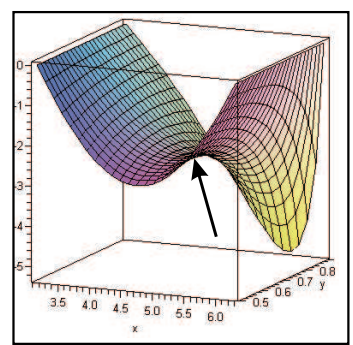

a)

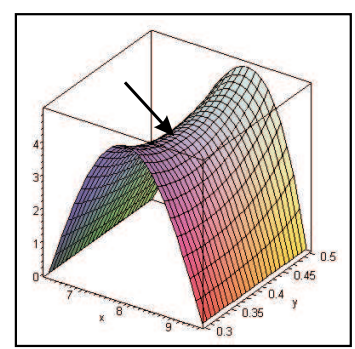

b)

Figure 4. Function $\gamma_{1+}(x, \xi)$.

$$
\gamma_{1+}(x ; \xi)=\frac{x \sin x}{1-\cos (\xi x)}=g(x ; \xi) \sin (x), \quad \text { where } g(x ; \xi):=\frac{x}{1-\cos (\xi x)} .
$$

Graphics of the functions $\gamma_{1+}(x ; \xi)$ and $\pm g(x ; \xi)$ for various parameter $\xi$ values are shown in Fig. 5. As we can see, graphics of the function $\gamma_{1+}(x ; \xi)$ oscillates between functions $g(x ; \xi)$ and $-g(x ; \xi)$.

Let us define function $\bar{g}(x ; \xi):=\frac{1}{1-\cos (\xi x)} \geq \frac{1}{2}$. The first two poles of this function are $x=\frac{2 \pi}{\xi}$ and $x=\frac{4 \pi}{\xi}$. When $x \geq \frac{4 \pi}{\xi}$ then $g(x ; \xi)=x \bar{g}(x ; \xi) \geq 2 \pi>\gamma_{3}$. So, in this case, the values of the function $\gamma_{1+}(x ; \xi)$ in extremum points are out the interval $\left[\gamma_{2}, \gamma_{3}\right]$.

Since

$$
g^{\prime}(x)=\frac{1-\cos (\xi x)-\xi x \sin (\xi x)}{(1-\cos (\xi x))^{2}},
$$

minimum points of function $g(x)$ are $x_{\min }=\frac{z_{k}}{\xi}, k \in \mathbb{N}_{o}$, where $z_{k}$ are positive roots of equation $1-\cos z-z \sin z=0$. Points $x=\frac{z_{k}}{\xi}, k \in \mathbb{N}_{e}$ are poles.

When $x \in\left(\frac{2 \pi}{\xi}, \frac{4 \pi}{\xi}\right)$ function $g(x)$ has one minimum point and $g\left(\frac{z_{3}}{\xi}, \xi\right)=$ $\frac{1}{\xi} \frac{z_{3}}{1-\cos z_{3}}$. We have $g(x, \xi) \geq \frac{3 z_{3}}{1-\cos z_{3}}>\gamma_{3}$.

Therefore, we need to investigate function $\gamma_{1+}$ only in the first interval $\left(0, \frac{2 \pi}{\xi}\right)$. If $\xi \leq \frac{1}{2}$ then $g(x, \xi) \geq \frac{1}{\xi} \frac{z_{1}}{1-\cos z_{1}} \geq \frac{2 z_{1}}{1-\cos z_{1}}>\left|\gamma_{2}\right|$.

Function's $\gamma_{1+}(x, \xi)$ extremum points we can find from a system

$$
\left\{\begin{array}{l}
\frac{\partial \gamma_{1+}}{\partial x}=\frac{(\sin x+x \cos x)(1-\cos (\xi x))+\xi x \sin x \sin (\xi x)}{(1-\cos (\xi x))^{2}}=0, \\
\frac{\partial \gamma_{1+}}{\partial \xi}=-\frac{\xi x \sin x \sin (\xi x)}{(1-\cos (\xi x))^{2}}=0 .
\end{array}\right.
$$

This system is equivalent

$$
\left\{\begin{array}{l}
\sin x+x \cos x=0, \\
\sin (\xi x)=0 .
\end{array}\right.
$$

For $\xi>\frac{1}{2}$ function $\gamma_{1+}(x ; \xi)$ has one local minimum when $x \in(\pi, 2 \pi)$. Then $\xi x \in\left(\frac{\pi}{2}, 2 \pi\right)$, i.e. we have only one extreme point $\left(x_{2}, \xi_{2}\right)$ and this point is saddle (see, Fig. 4 a) $\xi \in[0.5,0.9], x \in(\pi, 2 \pi)$ ) and $\gamma_{1+}\left(x_{2}, \xi_{2}\right)=\gamma_{2}$. 


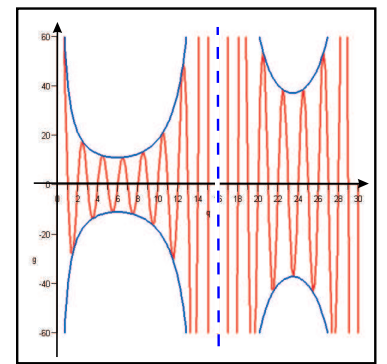

$\xi=\frac{1}{8}$

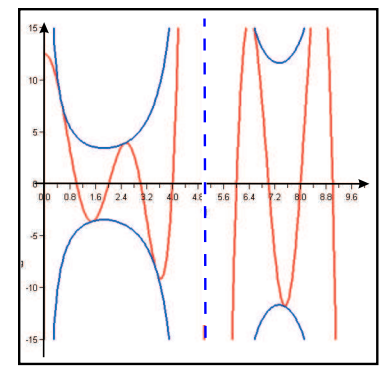

$\xi=\frac{2}{5}$

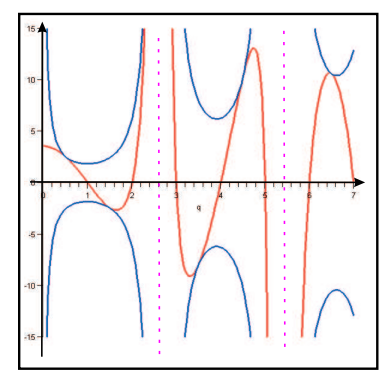

$\xi=\frac{3}{4}$

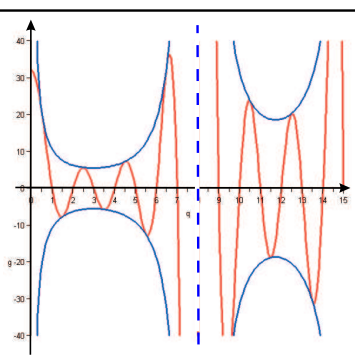

$\xi=\frac{1}{4}$

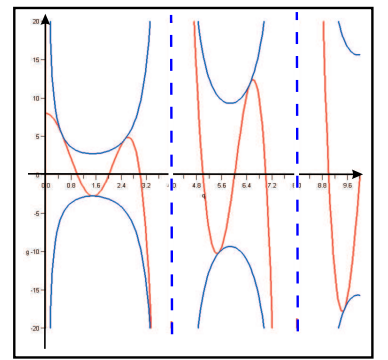

$\xi=\frac{1}{2}$

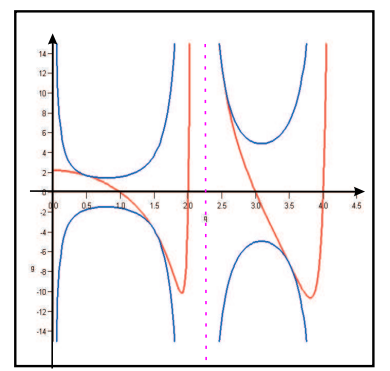

$\xi=\frac{95}{100}$

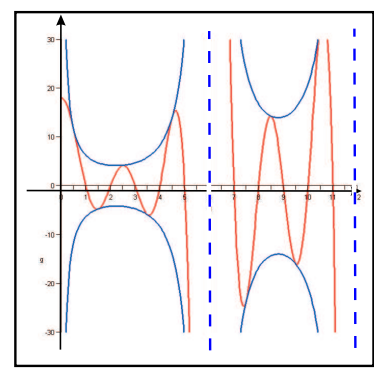

$\xi=\frac{1}{3}$
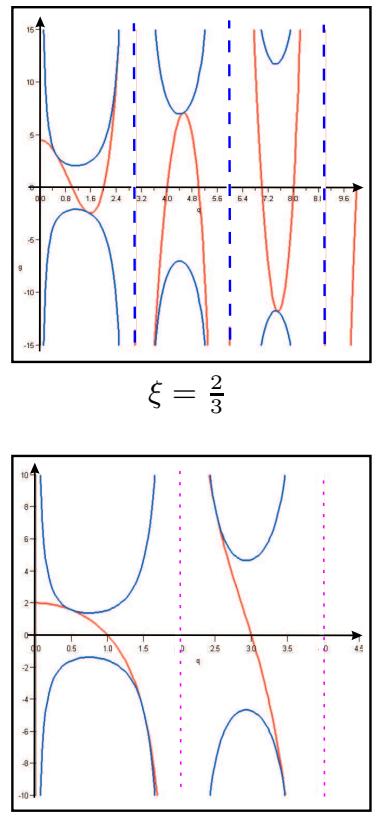

$\xi=1$

Figure 5. Functions $\gamma_{+}(x / \pi ; \xi)$ and $\pm g(x / \pi ; \xi)$.

If $\xi \leq \frac{1}{3}$ then $g(x ; \xi) \geq \frac{z_{3}}{1-\cos z_{3}}>\gamma_{3}$. If $\xi \geq \frac{2}{3}$ function $\gamma_{1+}(x ; \xi)$ hasn't local maximum points in the interval $\left(0, \frac{2 \pi}{\xi}\right)$. If $\frac{1}{2} \leq \xi<\frac{2}{3}$ then we have only one local maximum point $x_{\max }$ and

$$
\gamma_{1+}\left(x_{\text {max }}, \xi\right) \geq g\left(\frac{5 \pi}{2}, \xi\right) \geq 5 \pi /\left(2-2 \cos \left(\frac{5 \pi \xi}{2}\right)\right) \geq 5 \pi /\left(2-2 \cos \left(\frac{5 \pi}{2}\right)\right)>\gamma_{3} .
$$

If $\frac{1}{3} \leq \xi<\frac{2}{5}$ then we have two local maximum points $x_{\max }, x_{\max }^{\prime}$ and

$$
\gamma_{1+}\left(x_{\text {max }}^{\prime}, \xi\right) \geq g\left(\frac{9 \pi}{2}, \xi\right) \geq 9 \pi /\left(2-2 \cos \left(\frac{9 \pi \xi}{2}\right)\right) \geq 9 \pi /\left(2-2 \cos \left(\frac{9 \pi}{2}\right)\right)>\gamma_{3} .
$$

If $\frac{2}{5} \leq \xi<\frac{1}{2}$ then we have one local maximum point $x_{\max }$. Since $x_{\max } \in(2 \pi, 3 \pi)$, then $\xi x_{\max } \in(2 \pi / 3,3 \pi / 2)$ for $\xi \in(1 / 3,1 / 2)$, i.e. we have only one extreme point 
$\left(x_{3}, \xi_{3}\right)$ and this point is saddle (see, Fig. 4 b) $\xi \in[0.3,0.5], x \in(2 \pi, 3 \pi)$ ) and $\gamma_{1+}\left(x_{3}, \xi_{3}\right)=\gamma_{3}$.

So, for $\gamma_{2} \leq \gamma \leq \gamma_{3}$ horizontal line $\gamma$ intersects a graphic of the function $\gamma_{1+}$. If $\gamma=0$, then we get classical case with all positive and simple eigenvalues. When $\gamma_{2}<\gamma<\gamma_{3}$ all eigenvalues remain real and simple. We can enumerate as in classical case.

We have one negative eigenvalue for $\gamma>\frac{2}{\xi^{2}}$. So, all eigenvalues will be positive for all $\xi \in(0,1)$ if $\gamma \leq 2$.

Remark 10. When $\xi=\xi_{2}$ and $\gamma=\gamma_{2}$ or $\xi=\xi_{3}$ and $\gamma=\gamma_{3}$ then we have one multiple eigenvalue.

Remark 11. When $\xi=\xi_{0} \approx 0.55$ then $\gamma_{M}=\gamma_{1+}\left(x_{\max }\right)=\gamma_{1+}(0)=\frac{2}{\xi^{2}} \approx 6.599$ and all eigenvalues are positive and simple when $\gamma_{m}<\gamma<\gamma_{M}, \gamma_{m} \approx-2.55$.

\section{Conclusions}

- Sturm-Liouville problems (2.1)-(2.3) (Case 1) and (2.1)-(2.2), (2.4) (Case 2) have similar spectrum properties in the complex plane. Both spectrums haven't constant eigenvalues for irrational $\xi$ and countable number of non constant and constant eigenvalues for rational $\xi$. All constant eigenvalues are real positive numbers.

- Both problems have only one negative eigenvalue for $\gamma>\gamma_{0}$.

- Positive parts of spectrums are different for real $\gamma$ case. Problem in Case 2 has only real eigenvalues. In Case 1 problem has all real eigenvalues only for $\gamma_{m}(\xi) \leq \gamma \leq \gamma_{M}(\xi)$, but exists interval $\left[\gamma_{2}, \gamma_{3}\right] \subset\left[\gamma_{m}, \gamma_{M}\right]$ the same for all $\xi$. So, in this case for some real $\gamma$ multiple and complex eigenvalues exist.

\section{References}

[1] A.V. Bitsadze and A.A. Samarskii. Some elementary generalizations of linear elliptic boundary value problems. Dokl. Akad. Nauk SSSR, 185, 739-740, 1969.

[2] A. Boucherif. Differential equations with nonlocal boundary conditions. Nonlinear Analysis, 47, 2419-2430, 2001.

[3] A. Bouziani. On a class of parabolic equations with nonlocal boundary conditions. Bulletin de la Classe des Sciences, Académie Royale de Belgique, 61-77, 1999.

[4] J.R. Cannon. The solution of the heat equation subject to specification of energy. Quart. Appl. Math., 21(2), 155-160, 1963.

[5] D. Cao and R. Ma. Positive solutions to a second order multi-point boundary value problem. Electronic Journal of Differential Equations, 65, 1-8, 2000.

[6] R. Čiegis, A. Štikonas, O. Štikonienè and O. Suboč. Stationary problems with nonlocal boundary conditions. Mathematical Modelling and Analysis, 6(2), 178-191, 2001.

[7] R. Čiegis, A. Štikonas, O. Štikonienè and O. Suboč. A monotonic finite-difference scheme for a parabolic problem with nonlocal conditions. Differential Equations, 38(7), 1027-1037, 2002. 
[8] R. Čiupaila, Ž. Jesevičiūtė and M. Sapagovas. Eigenvalue problem for ordinary differential operator subject to integer condition. Nonlinear Analysis: Modelling and Control, 9(2), 109-116, 2004.

[9] L. Greenberg and M. Marletta. Numerical solution of non-self-adjoint Sturm-Liouville problems and related systems. SIAM Journal on Numerical Analysis, 38(6), 1800-1845, 2001.

[10] V.A. Ilyin and E.I. Moiseev. Nonlocal boundary value problem of the first kind for a Sturm-Liouville operator in its differential and finite difference aspects. Differential Equations, 23(7), 803-810, 1987. (in Russian)

[11] G. Infante. Eigenvalues of some non-local boundary-value problems. Proceedings of the Edinburgh Mathematical Society, 46, 75-86, 2003.

[12] N.I. Ionkin. The solution of a certain boundary value problem of the theory of heat conduction with a nonclassical boundary condition. Diff. Equations, 2(13), 294-304, 1977. (in Russian)

[13] N.I. Ionkin and E.A. Valikova. On eigenvalues and eigenfunctions of a non-classical boundary value problem. Math. Modelling, Russia, 1(8), 53-63, 1996. (in Russian)

[14] R. Ma. Positive solutions for a nonlinear three-point boundary-value problem. Electronic J. of Differential equations, 34, 1-8, 1998.

[15] A.I. Markushevich. Theory of Functions of a Complex Variable. Chelsea Publishing Company, New York, 1977.

[16] S. Pečiulytè, O. Štikonienè and A. Štikonas. On the spectrum of one stationary problem with nonlocal integral type boundary condition. Liet. matem. rink., 44(spec. nr.), 655659, 2004. (in Lithuanian)

[17] S. Sagwon. Global existence and decreasing property of boundary values of solution to parabolic equations with nonlocal boundary conditions. Pacif. J. Math., 1(193), 219226, 2000.

[18] M.P. Sapagovas. The eigenvalues of some problem with a nonlocal condition. Diff. Equations, 7(38), 1020-1026, 2002. (in Russian)

[19] M.P. Sapagovas and A.D. Štikonas. On the structure of the spectrum of a differential operator with a nonlocal condition. Diff. Equations, 41(7), 1010-1018, 2005.

[20] Y.F. Yin. On nonlinear parabolic equations with nonlocal boundary conditions. Journal of Mathematical Analysis and Applications, 1(185), 161-174, 1994.

[21] N.I. Yurchuk. Mixed problem with an integral condition for certain parabolic equations. Differents. Uravn., 12(22), 2117-2126, 1986. 
Šturmo-Liuvilio uždavinys stacionariajam diferencialiniam operatoriui su ịvairaus tipo nelokaliosiomis kraštinèmis salygomis

S. Pečiulytė, O. Štikonienè, A. Štikonas

Šiame straipsnyje nagrinejjamas Šturmo-Liuvilio uždavinys su viena nelokaliaja integralinio tipo kraštine sąlyga. Pirmoje straipsnio dalyje tiriamas Šturmo-Liuvilio uždavinys su dvieju tipu integraline nelokaliaja sąlyga. Irodytos tikriniu funkcijų ir tikrinių reikšmių bendrosios savybės komplesinejje plokštumoje. Antroje dalyje plačiau ištirtas realiuju tikrinių reikšmiu atvejis. Straipsnyje nagrinèjama kaip Šturmo-Liuvilio uždavinio spektras priklauso nuo kraštinių sąlygu parametrų. Priklausomai nuo nelokaliujju kraštinių sąlygu parametrų, aprašytas kokybinis tikrinių reikšmių pasiskirstymas. 